\title{
Somatic growth in the first six months of life of infants exposed to maternal smoking in pregnancy
}

\author{
Mariana Lopes de Brito ${ }^{1,4^{*}}$, Marina Nunes ${ }^{1,4}$, Juliana Rombaldi Bernardi ${ }^{1,2,4}$, Vera Lúcia Bosa ${ }^{2,3,4}$, \\ Marcelo Zubaran Goldani ${ }^{1,3,4}$ and Clécio Homrich da Silva ${ }^{1,3,4}$
}

\begin{abstract}
Background: Some studies suggest a relationship between maternal smoking during pregnancy and not only intrauterine fetal growth restriction or low birth weight, but also with changes in the postnatal growth and development. The objective of the present study was to investigate the effects of smoking during pregnancy on infants growth in the first 6 months of life compared with a control group and a group with idiopathic intrauterine growth restriction.

Methods: Longitudinal observational study using a convenience sample of newborns divided into three groups: infants of smoking mothers (tobacco), with idiopathic intrauterine growth restriction (IUGR) and a control group. The sample was selected from two hospitals in Porto Alegre, located in southern Brazil, between 2011 and 2015. Newborns were evaluated at birth, 7 and 15 days, and in the first, third, and sixth month. Anthropometric measures were weight, length and head circumference. The growth indicators used were expressed as z-scores. The analyses were performed using the generalized estimating equation method.
\end{abstract}

Results: The sample included 273 mother/newborn pairs: 86 tobacco group, 34 IUGR group, and 153 control group. In terms of weight at birth, all groups differed significantly $(p<0.001)$. The birth length of tobacco and control groups were similar, but the IUGR group was lower than both $(p<0.001)$. We found no differences in growth trajectory between tobacco and control group, but there were differences in the growth of the IUGR group when compared with the other groups. At 6 months of age, all groups had similar anthropometric measurements.

Conclusion: Intrauterine growth restriction had major impact on the growth trajectory of the infants studied, regardless of other factors, such as smoking and diet.

Keywords: Pregnancy, Smoking, Intrauterine growth restriction, Infants, Growth, Longitudinal studies

\section{Background}

Because of its high prevalence obesity has become a worldwide epidemic in recent years. The association of maternal smoking with the development of childhood obesity has been demonstrated in several studies. Two meta-analyses of observational studies in population of 333 months reported odds ratios of approximately 1.5 for overweight in the children of smoking mothers $[1,2]$. In

\footnotetext{
* Correspondence: mariana.lopesdebrito@gmail.com

${ }^{1}$ Graduate Program in Child and Adolescent Health, Universidade Federal do Rio Grande do Sul (UFRGS), Porto Alegre, Brazil

${ }^{4}$ Center for the Study of Child and Adolescent Health, Porto Alegre, Brazil

Full list of author information is available at the end of the article
}

general, about $40 \%$ of the children in the world are exposed to smoking in the home. Environmental exposures occurring within a critical window of development, such as pregnancy or lactation, may trigger permanent changes to the metabolism, leading to diseases in adulthood, a phenomenon currently called programming [3].

It is estimated that 250 million women smoke on a daily basis worldwide. Despite the well-known harm many women do not quit smoking during pregnancy. In pregnant women, the nicotine and carbon monoxide components of cigarette smoke may cause damage to both the mother and the fetus, crossing the placental barrier [4]. 
Smoking during pregnancy is one of the greatest public health problems in the world due to its high prevalence and deleterious consequences. Smoking leads the preventable causes of unfavorable pregnancy outcomes and increased risk of morbidity and mortality in newborns $[5,6]$. In Brazil, the prevalence of smoking during pregnancy ranges from 23 to $25 \%$. The main maternal risk factors are Caucasian race, low socioeconomic status, low educational level, and single marital status [7].

Smoking has been associated with shorter duration of pregnancy miscarriages, and shorter duration of breastfeeding. During pregnancy, smoking is also a risk factor for intrauterine growth restriction (IUGR), perinatal mortality, low birth weight (LBW), and neurological abnormalities [8].

Several studies have shown an association between smoking during pregnancy and the development of obesity and hypertension in childhood and adolescence. However there is less consistent evidence concerning the association of maternal smoking during pregnancy and postnatal growth. Some authors have demonstrated the potential effect of tobacco smoke exposure and the development of childhood obesity [9-11]. More recently, other authors have shown the presence of stunting in children exposed to tobacco smoke during the intrauterine period [12-14].

The objective of the present study was to investigate the effects of smoking during pregnancy on infants' growth in the first 6 months of life compared with a control group and a group with idiopathic intrauterine growth restriction.

\section{Methods}

This is a longitudinal observational study using a convenience sample of mothers and their newborns divided into groups according to maternal exposures occurring during pregnancy. These groups were observed during the first semester of their children's lives.

\section{Participants}

The sample was selected from the Hospital de Clínicas de Porto Alegre and Hospital Femina of the Grupo Hospitalar Conceição located in the city of Porto Alegre, capital city of the state of Rio Grande do Sul (Brazil), from September 2011 to August 2015. The information about gestational age was collected from medical hospital records through of last menstrual period and precocious ultrasonography. There were included only pregnancy to term in this study. The mother/newborn pairs were divided into three groups: 1) smoking mothers (tobacco group): mothers who reported they smoked during pregnancy, regardless of the duration of exposure or the number of cigarettes smoked; 2) newborns who had idiopathic intrauterine growth restriction (IUGR group): newborns who had a birth weight below the 5th percentile according to the fetal growth curve proposed by Alexander et al. [15] and 3) control group: included nonsmoking mothers without diseases and those whose newborns did not have IUGR. These pairs did not have any diseases, liked gestational diabetes and hypertension, lived in the city of Porto Alegre, and the infants were delivered at the hospitals participating in the study. We excluded mothers who tested positive for HIV (Human Immunodeficiency Virus), twin infants, and newborns who require hospitalization and/or with acute diseases and/or congenital birth defects.

The use of an IUGR group may be considered as a second control group because generally children exposed to maternal smoking have lower birth weight and length. The combination of weight to be recovered and persistent height deficits could result in higher body mass index in children of smoking mothers [16].

\section{Data collection}

The mother/newborn pairs were evaluated at birth 7 and 15 days, and in the first, third, and sixth month of life. A detailed description of the methods used in the present study has been published previously [17]. The anthropometric measures taken in all medical visits were weight, length, and head circumference. The following indicators were expressed as z-scores according to the WHO (World Health Organization) growth curves [18]: weight-for-age, weight-forlength, length/height-for-age and head circumference-for-age.

\section{Diet}

The type of food given to the child was recorded using a 24-h diet recall and a questionnaire about the introduction of food. The instruments were administered in all interviews.

\section{Anthropometric data}

Two investigators previously trained were taken the anthropometric measures. They used standardized techniques and calibrated equipment with the purpose of reducing interobserver and intraobserver variability. Infants' body weight was measured in kilograms using a portable digital electronic scale (Marte ${ }^{\bullet}$ Scientific, São Paulo, Brazil) that was accurate to within $50 \mathrm{~g}$. Each infant's weight was then calculated by subtracting the mother's weight from the total weight of mother and child combined. Infants' length was measured in supine position, on a flat and stable surface, such as a table, using a portable stadiometer (Alturexata ${ }^{\oplus}$, Belo Horizonte, Brazil). Children's head circumference was measured around the largest occipital-frontal diameter across the forehead, just above the ears, using a non-stretch tape measure (Lange ${ }^{\varpi}$, Ann Arbor, United States). 


\section{Statistical analysis}

The primary outcome we analyzed consisted of growth in the first 6 months of life. Our results were described and expressed as mean and standard deviation to evaluate the distribution of variables. The KolmogorovSmirnov test was used to assess normality. The chisquare test was used to assess the associations between categorical variables; whereas the $t$-test was used for parametric continuous variables. We used the generalized estimated equation (GEE) method which is based on the generalized linear model, to assess the growth trajectory of each group during the follow-up period and after, the Bonferroni multiple comparison test was used.

The significance level was set at $5 \%$ and the statistical analyses were performed using the Statistical Package for the Social Sciences (SPSS), version 18.0.

\section{Ethical aspects}

The project was submitted to the Research Ethics Committee at the Hospital de Clínicas de Porto Alegre and Grupo Hospitalar Conceição. The project was approved under the protocol numbers 110097 and 11027 respectively.

\section{Results}

Data collection was conducted between September 2011 and August 2015. Our sample included 273 mother/ newborn pairs. Of these 34 pairs were allocated to the IUGR group, 86 pairs were included in the tobacco group, and 153 pairs formed the control group.

The distribution of sociodemographic perinatal, and anthropometric variables between groups is shown in Table 1. The education level of tobacco group was significantly lower than the other groups $(p=0.036)$. In terms of weight at birth, all groups differed significantly $(p<0.001)$. There was no difference between the tobacco group and the control group, but the weight of the IUGR group was lower than the groups $(p<0.001)$, regarding length and head circumference.

The average consumption and the median of cigarettes a day was respectively 10 and 11 cigarettes in the tobacco group. These similar values show that the dose consumed was similar among individuals in the group. Additionally, a national survey found that the average number of cigarettes consumed by the Brazilian is 12.9 cigarettes per day [19]. The sociodemographic characteristics also make us believe that consumption was similar, a time statistically significant differences were no found.

The weight-for-age indicator is shown in Fig. 1 where it was observed a statistically significant difference between the three groups-intergroups $(p<0.001)$-and when the measures were taken-intragroups $(p<0.001)$. At birth, all groups differed significantly in mean $\mathrm{z}$ score. This difference persisted until the fifteenth day. At 1 and 3 month only the IUGR group differed. In the sixth month, the IUGR group only differed the control group $(p=0.03)$.

The length/height-for-age indicator is shown in Fig. 2 where we demonstrated a statistically significant difference intergroups $(p<0.001)$ and intragroups $(p<0.001)$. At birth, the infants in the IUGR group differed from those other groups $(p<0.001)$, showing lower mean $\mathrm{z}$-score. At 7 and 15 days the IUGR and tobacco group differed from control group. At 1 month, all groups differed statistically significantly. At 3 months, the IUGR and control group showed differences $(p=0.00)$. There was no difference between the groups at 6 months of age.

The weight-for-length indicator is shown in Fig. 3 where the IUGR group differed from the others at birth. At 7 days all groups differed statistically significantly. At 15 days the IUGR and tobacco group differed from control group. After that, there was no difference of the measures between the groups.

The head circumference-for-age indicator is shown in Fig. 4 where the newborns of the IUGR group differed from those of the tobacco group $(p<0.001)$ and the control group $(p<0.001)$. At 7 days, all groups differed statistically significantly. At 15 days the IUGR group and the tobacco group showed similar measures; however these groups differed from the control group. At 1, 3 and 6 months, there was no difference between the groups.

In terms of diet during the first 6 months of life we found that the frequency of exclusive breastfeeding decreased during the follow-up period similarly in the three groups. Conversely, the artificial feeding increased with age. The introduction of solid or semisolid foods occurred similarly in the tobacco group and the control group after 15 days of life. This variables did not show statistically significant differences between the groups $(p>0.05)$.

\section{Discussion}

The present study showed that the growth trajectory of children exposed to maternal smoking during pregnancy was similar to the control group. However as well as other studies, newborns exposed to tobacco showed differences in the weight-for-age at birth when compared with the control group, presenting a mean reduction of $314 \mathrm{~g}$ in birth weight.

In a study conducted by Suzuki et al. [20] the authors found that maternal smoking was a significant predictor of LBW in both genders, thus increasing the risk by 3.2 times. Similarly, Vardavas et al. [21] reported that smoking during pregnancy was associated with a reduction between 120 and $150 \mathrm{~g}$ in birth weight.

Epidemiological studies have shown that adverse environmental factors during pregnancy may cause IUGR and LBW thus leading to the potential development of metabolic syndrome. Anthropometric measures, such as birth weight, are influenced by genetic and epigenetic factors 
Table 1 Maternal sociodemographic, perinatal, and anthropometric characteristics according to group. IVAPSA cohort, Porto Alegre, September 2011-August 2015

\begin{tabular}{|c|c|c|c|c|c|c|c|c|}
\hline & $\begin{array}{l}\text { IUGR } \\
\left(34^{* *}\right)\end{array}$ & & $\begin{array}{l}\text { TOBACCO } \\
\left(86^{* *}\right)\end{array}$ & & $\begin{array}{l}\text { CONTROL } \\
\left(153^{* *}\right)\end{array}$ & & $\begin{array}{l}\text { TOTAL } \\
\text { (273) }\end{array}$ & \\
\hline \multicolumn{9}{|l|}{ Sociodemographic } \\
\hline \multicolumn{9}{|l|}{ Ethnicity, n (\%) } \\
\hline White & 16 & $(47.1)$ & 50 & $(58.1)$ & 92 & $(60.1)$ & 158 & $(57.9)$ \\
\hline Black & 12 & $(35.3)$ & 22 & $(25.6)$ & 32 & $(20.9)$ & 66 & $(24.2)$ \\
\hline Others $^{a}$ & 6 & $(17.6)$ & 14 & $(16.3)$ & 29 & $(19.0)$ & 49 & $(18.0)$ \\
\hline \multicolumn{9}{|l|}{ Marital status, n (\%) } \\
\hline Living with a partner & 29 & $(85.3)$ & 58 & $(67.4)$ & 129 & $(84.3)$ & 216 & $(79.1)$ \\
\hline Single & 5 & $(14.7)$ & 28 & $(32.6)$ & 24 & $(15.7)$ & 57 & $(20.9)$ \\
\hline \multicolumn{9}{|l|}{ Educational level, n (\%) } \\
\hline$\leq 8$ years & 9 & $(26.5)$ & 43 & $(51.2)$ & 49 & $(32.9)$ & 101 & $(37.8)$ \\
\hline $9<11$ years & 22 & $(64.7)$ & 38 & $(45.2)$ & 89 & $(59.7)$ & 149 & $(55.8)$ \\
\hline$\geq 12$ years & 3 & $(8.80)$ & 3 & $(3.60)$ & 11 & $(7.40)$ & 17 & $(6.40)$ \\
\hline \multicolumn{9}{|l|}{ Social class, n (\%) } \\
\hline Hight & 10 & (31.3) & 21 & $(25.9)^{b}$ & 60 & $(41.4)$ & 59 & $(31.7)$ \\
\hline Middle & 17 & $(53.1)$ & 52 & $(64.2)$ & 73 & $(50.3)$ & 106 & $(57.0)$ \\
\hline Low & 5 & $(15.6)$ & 8 & (9.9) & 12 & $(8.30)$ & 21 & $(11.3)$ \\
\hline Age, $x \pm S D$ & 23.79 & $(7.07)$ & 24.29 & $(6.24)$ & 25.79 & $(6.79)$ & 25.06 & $(6.79)$ \\
\hline \multicolumn{9}{|l|}{ Perinatal } \\
\hline \multicolumn{9}{|l|}{ Mode of delivery, n (\%) } \\
\hline Cesarean section & 12 & $(35.3)$ & 21 & $(24.4)$ & 45 & $(29.6)$ & 78 & $(28.7)$ \\
\hline Vaginal delivery & 22 & $(64.7)$ & 65 & $(75.6)$ & 107 & $(70.4)$ & 194 & $(71.3)$ \\
\hline \multicolumn{9}{|l|}{ Child gender, n (\%) } \\
\hline Female & 20 & $(58.8)$ & 42 & $(48.8)$ & 86 & $(56.2)$ & 148 & $(54.2)$ \\
\hline Male & 14 & $(41.2)$ & 44 & $(51.2)$ & 67 & $(43.8)$ & 125 & $(45.8)$ \\
\hline Mean Apgar-5 min, $x( \pm S D)$ & 9.64 & $(0.48)$ & 9.52 & $(0.62)$ & 9.46 & $(0.59)$ & 9.50 & $(0.59)$ \\
\hline Mean weight-grams $( \pm S D)$ & 2521.35 & $(29.42)$ & 3071.45 & $(57.02)$ & 3385.59 & $(36.78)$ & $3179.39^{*}$ & $(32.50)$ \\
\hline Mean length-cm $( \pm \mathrm{SD})$ & 46.121 & $(0.28)$ & 48.012 & $(0.24)$ & 49.306 & $(0.16)$ & $48.511^{*}$ & $(0.14)$ \\
\hline Mean head circumference-cm $( \pm S D)$ & 31.922 & $(0.21)$ & 33.717 & $(0.14)$ & 34.060 & $(0.12)$ & $33.695^{*}$ & $(0.09)$ \\
\hline \multicolumn{9}{|l|}{ Anthropometric } \\
\hline \multicolumn{9}{|l|}{ Pre-pregnancy BMI, n (\%) } \\
\hline Underweight $\left(<18.5 \mathrm{Kg} / \mathrm{m}^{2}\right)$ & 0 & $(0.00)$ & 1 & $(2.10)$ & 2 & $(1.90)$ & 3 & $(1.70)$ \\
\hline Normal weight $\left(\geq 8.5 \leq 24.9 \mathrm{Kg} / \mathrm{m}^{2}\right.$ ) & 19 & $(76.0)$ & 25 & $(52.1)$ & 62 & $(58.5)$ & 106 & $(59.2)$ \\
\hline Overweight $\left(>24.9 \leq 29.9 \mathrm{Kg} / \mathrm{m}^{2}\right)$ & 2 & $(8.00)$ & 13 & $(27.1)$ & 29 & $(27.4)$ & 44 & $(24.6)$ \\
\hline Obese $\left(>29.9 \mathrm{Kg} / \mathrm{m}^{2}\right)$ & 4 & $(16.0)$ & 9 & $(18.8)$ & 13 & $(12.3)$ & 26 & $(14.5)$ \\
\hline Gestational weight gain (Kg) (SD) & 11.53 & $(5.80)$ & 14.00 & (6.89) & 13.41 & $(6.71)$ & 13.36 & $(6.68)$ \\
\hline
\end{tabular}

athers: asian, brown and native brasilian

${ }^{\mathrm{b}}$ The education level of tobacco group was significantly lower than the other groups $(p=0.036)$

* The perinatal variables birth weight, birth length, and head circumference at birth were statistically significant $(p<0.001)$. The other variables did not show statistically difference between the groups $(p>0.05$ in the chi-square test for categorical variables and Student's $t$ test for parametric continuous variables)

${ }^{* *}$ Number (proportion) of missing data: Educational level $n=6(2,19 \%)$, social class $n=15(5.5 \%)$, mode of delivery $n=1(0.36 \%)$, Mean apgar 5 min $n=3(1,09)$, Mean weight $n=1(0,36)$, Mean length $n=4(1,46)$, Mean head circumference $n=7(2,56)$, pre-pregnancy BMI $n=94(34,43 \%)$, and gestational weight gain $n=38(13.91 \%)$

combined with the intrauterine environment [22]. It is possible to assume that the relationship between smoking during pregnancy and overweight in childhood may be mainly related to IUGR and LBW. Such hypothesis is based on the fact that, in the present study, it was observed that newborns in tobacco group did not show 


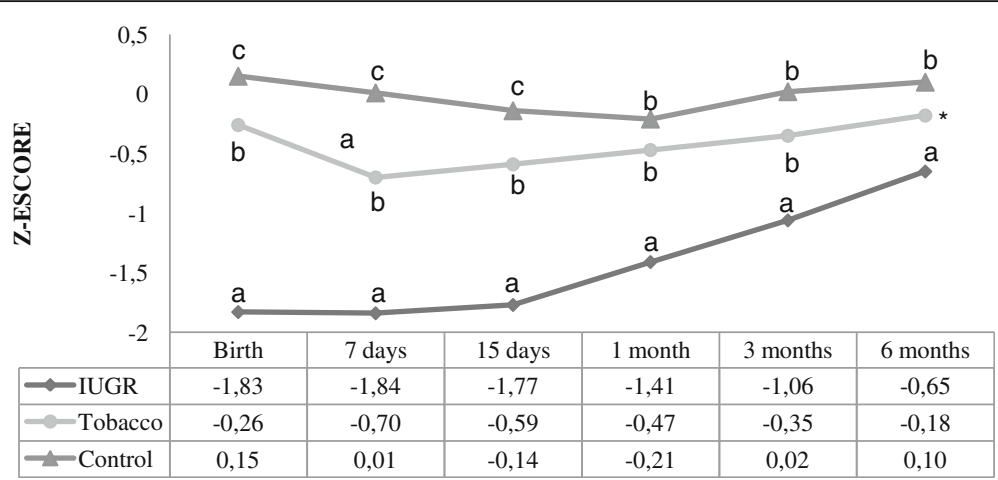

Fig. 1 Weight-for-age $z$-score. Analysis performed using the generalized estimated equation (GEE) model. - Comparison between the groups ( $P<0.001$ ), comparison between times $(P<0.001$ ), and comparison between group and times (interaction: $P<0.001$ ). - We used letters $\mathrm{a}$, $\mathrm{b}$, and $\mathrm{c}$ to express the differences between the three groups. Means followed by the same letter showed no statistically significant difference $(P>0.05)$ based on the GEE analysis, followed by the Bonferroni multiple comparison test. * There was no statistically significant difference between the tobacco group and the other groups

LBW and their growth trajectory was similar to controls despite their exposure to tobacco smoke.

Despite others studies in children and adults had showed the association between of maternal smoking in pregnancy with overweight in adulthood even adjustment for birth weight [23, 24], some publications have suggested that low birth weight has an important role in the association of fetal nicotine exposure and offspring's overweight $[25,26]$.

Regarding the length/height-for-age indicator the IUGR group was different from the others at birth. At 7 and 15 days, the IUGR and tobacco were similar. However, the difference between groups disappeared after 6 months. A study conducted in the city of Pelotas, southern Brazil, found that maternal smoking during pregnancy adversely affected children's height during early childhood, childhood, and adolescence [12]. Some mediators like Insulin, insulin- like growth factor (IGF), insulin-like growth factor binding protein-2 (IGFBP-3) show lower concentration in children of smoking mothers and, on the opposite, hemoglobin and erythropoietin have higher concentration. This could explain to fetal hypoxia produced by maternal smoking. These alterations might cause a negative effect throughout life, resulting in poor growth in childhood [13].

Conversely a cohort study conducted in England (ALSPAC) found differences in the growth patterns of children of smokers versus nonsmokers. Children of smoking mothers grew faster in early childhood, but more slowly in preschool years. However, the differences between the groups were minor and may be attributed to chance [14]. Our study found no difference in birth length between smoking and control group, both differ in the length/height-for-age only the seventh to 30 day of life. However, the difference of children in the tobacco

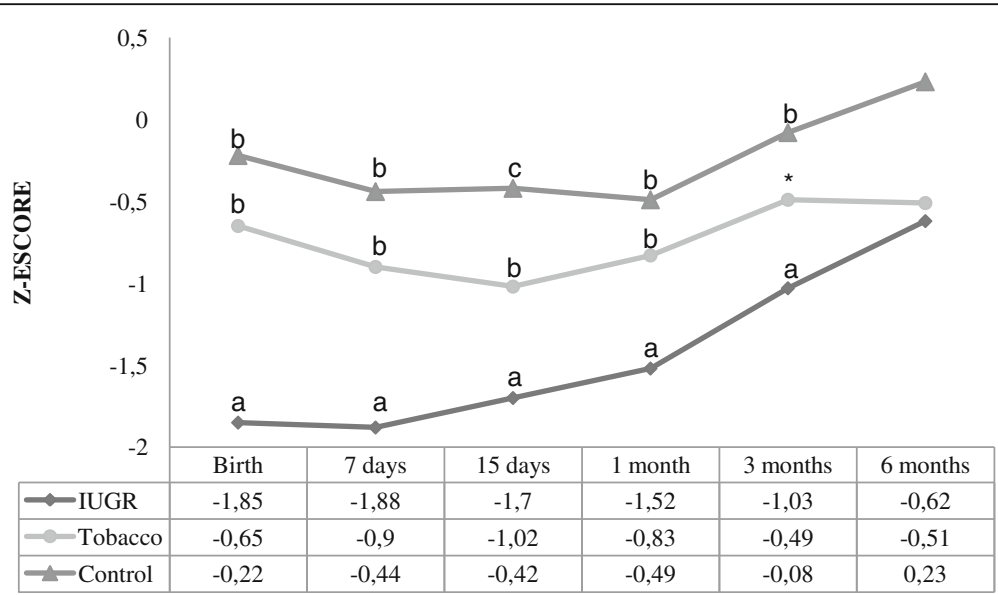

Fig. 2 Length/height-for-age z-score. Analysis performed using the generalized estimated equation (GEE) model. - Comparison between the groups $(P<0.001)$, comparison between times $(P<0.001)$, and comparison between group and times (interaction: $P<0.001)$. - We used letters a, $b$, and $c$ to express the differences between the three groups. Means followed by the same letter showed no statistically significant difference $(P>0.05)$ based on the GEE analysis, followed by the Bonferroni multiple comparison test. ${ }^{*}$ There was no statistically significant difference between the tobacco group and the other groups 


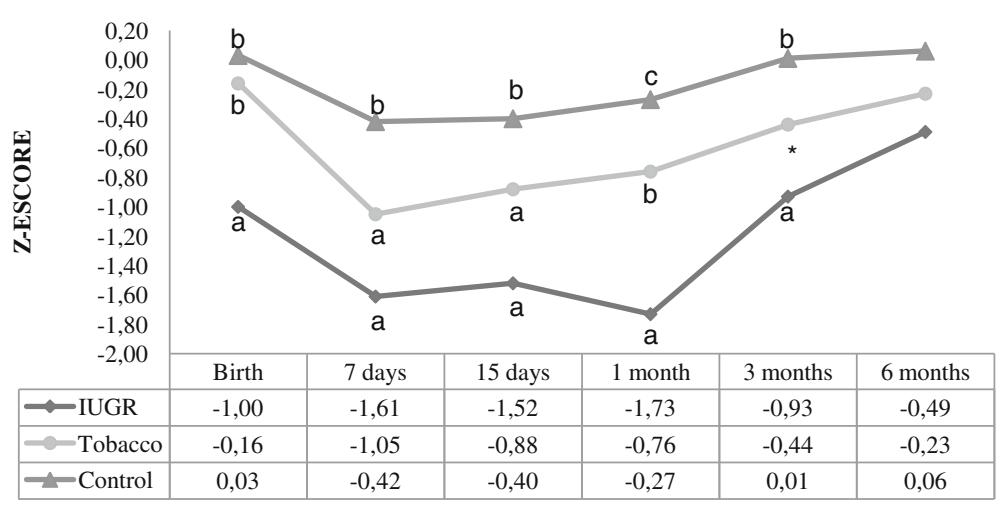

Fig. 3 Weight-for-length $z$-score. Analysis performed using the generalized estimated equation (GEE) model. - Comparison between the groups ( $P<0.001$ ), comparison between times $(P<0.001$ ), and comparison between group and times (interaction: $P<0.001)$. - We used letters a, b, and $\mathrm{c}$ to express the differences between the three groups. Means followed by the same letter showed no statistically significant difference $(P>0.05)$ based on the GEE analysis, followed by the Bonferroni multiple comparison test

group disappeared at 30 days of life, despite having lower birth weight compared with the control group.

In agreement with these findings another study found that the association between maternal smoking during pregnancy and overweight in male young adults could be explained in part by the parents' socioeconomic status and by family factors that were not fully measured, such as the eating habits of the family [27].

About scholar education in our study, pregnant women of tobacco group had less education, a result similar to other studies [28]. In the United States, women with 12 or less years of education were more than 3 times as likely to smoke during pregnancy as women with more than 12 years of education [29].

The number of cigarettes smoked can influence the anthropometric measurements of infants since as some studies suggest, tobacco shows dose-response [30, 31]. In our study, pregnant women consumed an average of 10 cigarettes a day, which can be considered moderate, since most studies considered heavy smokers those with a consumption of 20 or more cigarettes/day [12, 32]. This may be one of the factors contributing to the growth trajectory of the group exposed to smoke was similar to the control.

The association between weight gain in childhood and later overweight could be partially explained by the type of diet. The use of infant formula is associated with more rapid weight gain [33]. Furthermore, breastfed infants gain weight more slowly. Breast milk production is stimulated by the suction of the baby, and therefore it would be unlikely that a rapid weight gain in breastfed infants is exclusively due to overfeeding [34]. Thus,

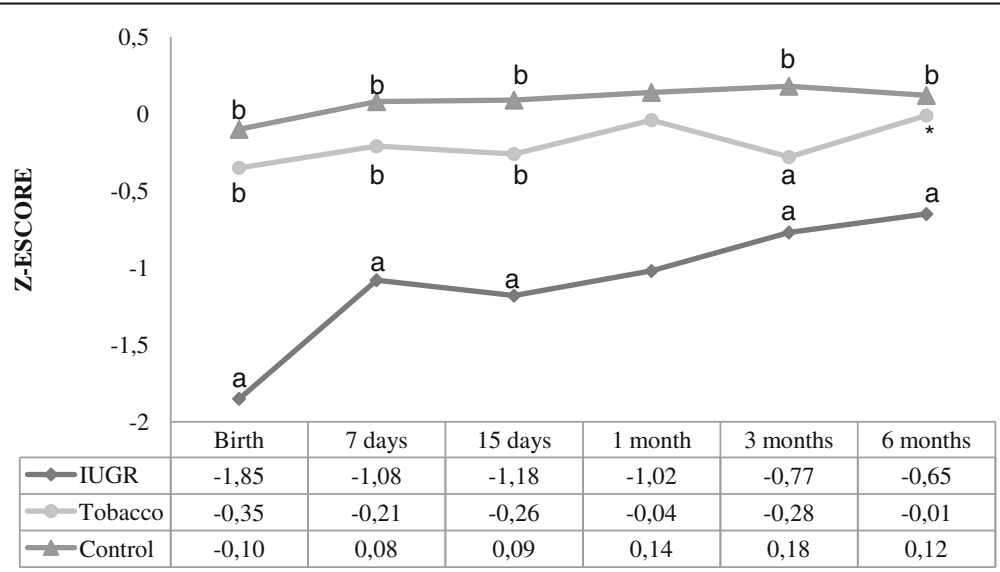

Fig. 4 Head circumference-for-age $z$-score. Analysis performed using the generalized estimated equation (GEE) model. - Comparison between the groups $(P<0.001)$, comparison between times $(P<0.001)$, and comparison between group and times (interaction: $P<0.001)$. - We used letters $a, b$, and $c$ to express the differences between the three groups. Means followed by the same letter showed no statistically significant difference $(P>0.05)$ based on the GEE analysis, followed by the Bonferroni multiple comparison test. *There was no statistically significant difference between the tobacco group and the other groups 
adjusting for the type of food is important to understand the association with weight gain. The groups showed no differences in the type of food received at each follow-up time point. All groups showed reduced prevalence of exclusive breastfeeding during the follow-up period, as well as early introduction of complementary foods.

In the IUGR group head circumference at birth was significantly lower in comparison with the other groups, remaining distinct until 3rd month of life. Several studies have found that children exposed to smoking during pregnancy have a lower head circumference at birth $[6$, 35]. However, Matijasevich et al. [16] found that the deficits observed at birth were not present during childhood. This finding is similar to what we found, since there was no difference between the groups at 6 months.

Following up the growth of head circumference at regular time intervals makes it possible to investigate whether brain development is appropriate as there is strong correlation between head circumference growth and brain development [36]. The growth curve shows the dynamics of the global growth of the skull and its internal structures. Therefore, serial measurements facilitate early recognition of deviations in the trajectory of head growth [37] and thus, it becomes possible to carry out early intervention.

The present study has several strengths. First six measures of growth from birth to 6 months of life collected. This continuous and close approach was important because it made it possible to identify potential critical or sensitive periods for intervention, which were based on patterns of biological growth and could be predictors of overweight or obesity in childhood. Second, the method for longitudinal data analysis using GEE allowed for the analysis of continuous outcomes, even when there is missing some information regarding certain participants of the study, thus making it possible to include all participants, which may avoid bias selection.

This study also had some limitations. It was not possible to stratify the group of tobacco in relation to the gestational period of exposure. Prenatal smoking results in overall neonatal growth restriction however, in mothers who stop smoking before late pregnancy, neonates will be phenotypically similar to those who were not exposed during pregnancy, possibly decreasing the likelihood of catch-up growth [30].

Although our study did not find differences in the growth patterns of children exposed to smoking in the first months of life it has been demonstrated that the perinatal period is a key period for the development of obesity [38]. Maternal smoking during pregnancy is one of the few modifiable risk factors in the prenatal period, being the leading cause of LBW and IUGR $[39,40]$. Maternal smoking may also contribute to the syndrome of sudden infant death and cause major changes in the development of the fetal nervous system [41].

Even though our findings showed no significant changes in the growth of children of smoking mothers do not rule out the possibility these effects arise at later ages. According to a study conducted in Germany, it's was found an association of maternal smoking during the pregnancy with overweight in children. This phenomenon became evident between 4 and 6 years and, afterwards, it was accentuated until the adolescence. This and other studies have shown that the effect of maternal smoking seems to influence the development of obesity in children with increasing age [42].

In short intrauterine growth restriction seems to have more impact on the growth trajectory of the infants studied, regardless of other factors, such as smoking and diet. Whereas that, infants exposed to maternal smoking during pregnancy born with adequate weight showed the same pattern of growth control group.

\section{Conclusion}

Considering the many adverse effects of smoking during pregnancy, women of reproductive age should be advised to stop smoking before trying to conceive, because quitting smoking after receiving the diagnosis of pregnancy may not be enough to protect their children from potential risks [43]. In addition, the harmful effects of tobacco are well known and do not apply only to somatic growth, as they affect other systems of a child's organism.

Therefore, anti-smoking campaigns should continue to be focused on periods of increased vulnerability of the life cycle, directly or indirectly involving mothers, children, and adolescents. In a similar manner, advertising restrictions should be maintained to reduce the prevalence of consumption and, particularly, to discourage young people from having their first experience with cigarettes and other tobacco related products.

\section{Abbreviations}

GEE: Generalized estimated equations; HIV: Human Immunodeficiency Virus; IGF: Insulin-like growth factor; IGFBP-3: Insulin-like growth factor binding protein-3; IUGR: Intrauterine growth restriction; LBW: Low birth weight; SPSS: Statistical Package for the Social Sciences; WHO: World Health Organization

\section{Acknowledgements}

The researchers of the IVAPSA Cohort: Tanara Voguel, Rafaela da Silveira, Priscyla Bones, Isabel Werlang, Thamíris Medeiros, Salete Matos, Sara Bruneto, Fabiana Copês, and Amanda Ferreira.

All the participants for their time and patience.

\section{Funding}

The research was supported by FAPERGS/CNPq 10/0018.3 (Conselho Nacional de Desenvolvimento Científico e Tecnológico), PRONEX 2009 (Programa de Apoio a Núcleos de Excelência), FIPE/HCPA (Fundo de Incentivo à Pesquisa e Eventos do Hospital de Clínicas de Porto Alegre) and CAPES (Comissão de Aperfeiçoamento de Pessoal do Nível Superior)-Brazil. 


\section{Availability of data and materials}

The data that support the findings of this study are available from the corresponding author upon reasonable and justified request. The researchers of the present project committed to preserve the privacy of the patients whose data were collected in charts and databases of the Hospital de Clínicas of Porto Alegre.

\section{Authors' contributions}

MLB participated in data collection, literature review, analysis and writing article. MN and JRB participated in the design of the study and performed the statistical analysis. VLB, MZG and CHS conceived of the study, and participated in its design and coordination and helped to draft the manuscript. All authors read and approved the final manuscript.

\section{Competing interests}

The authors declare that they have no competing interests.

\section{Consent for publication}

"Not applicable" in this section.

\section{Ethics approval and consent to participate}

This project was approved by the Ethics and Research Committees of the Hospital de Clínicas of Porto Alegre-HCPA (protocol n 11-0097) and the Grupo Hospitalar Conceição-GHC (protocol no. 11-027). Once the eligibility criteria for the project were met, the women were invited to participate and, after the signing of the informed consent form, they were included in the study. The consent term included the authorization for the participation of the children in this study.

\section{Author details}

'Graduate Program in Child and Adolescent Health, Universidade Federal do Rio Grande do Sul (UFRGS), Porto Alegre, Brazil. ${ }^{2}$ Departament of Nutrition, Universidade Federal do Rio Grande do Sul (UFRGS), Porto Alegre, Brazil. ${ }^{3}$ Department of Pediatrics, Hospital de Clínicas de Porto Alegre, Porto Alegre, Brazil. ${ }^{4}$ Center for the Study of Child and Adolescent Health, Porto Alegre, Brazil.

\section{Received: 26 November 2015 Accepted: 22 February 2017}

Published online: 09 March 2017

\section{References}

1. Ino $T$, et al. Maternal smoking during pregnancy and offspring obesity: Meta-analysis. Pediatr Int. 2010;52:94.

2. Oken E, Levitan EB, Gillman MW. Maternal smoking during pregnancy and child overweight: systematic review and meta-analysis. Int J Obes (Lond). 2008;32:201.

3. Lisboa PC, de Oliveira E, de Moura EG. Obesity and endocrine dysfunction programmed by maternal smoking in pregnancy and lactation. Front Physiol. 2012;3:437.

4. Ardalić D, et al. The Influence of Maternal Smoking Habits Before Pregnancy and Antioxidative Supplementation During Pregnancy on Oxidative Stress Status in a Non-Complicated Pregnancy. Adv Clin Exp Med. 2014;23:575.

5. Coleman-cowger VH. Smoking cessation intervention for pregnant women: a call for extension to the postpartum period. Matern Child Health J. 2012; 16:937.

6. Zhang $L$, et al. Maternal smoking during pregnancy and anthropometric measurements of newborns: a population-based study in southern of Brazil. Cad Saude Publica. 2011;27:1768.

7. Galão A, et al. Comparison between Postpartum Smokers and Ex-Smokers as to Breastfeeding Duration and its Impact on the Health of Newborns. Rev Bras Cancerol. 2011;57:379-85.

8. Einarson A, Riordan S. Smoking in pregnancy and lactation: a review of risks and cessation strategies. Eur J Clin Pharmacol. 2009;65:325.

9. Von kries $\mathrm{R}$, et al. Maternal smoking during pregnancy and childhood obesity. Am J Epidemiol. 2002;156:954-61.

10. Dubois L, Girard M. Early determinants of overweight at 4.5 years in a population-based longitudinal study. Int J Obes (Lond). 2006;30:610.

11. Power C, Jefferis BJ. Fetal environment and subsequent obesity: a study of maternal smoking. Int J Epidemiol. 2002;31:413.
12. Martínez-mesa J, et al. Life course association of maternal smoking during pregnancy and offspring's height: data from the 1993 Pelotas (Brazil) birth cohort. J Adolesc Health. 2012;51(6 Suppl):S53-7.

13. Leary S, Davey Smith G, Ness A. Smoking during pregnancy and components of stature in offspring. Am J Hum Biol. 2006;18:502.

14. Howe LD, et al. Maternal smoking during pregnancy and offspring trajectories of height and adiposity: comparing maternal and paternal associations. Int J Epidemiol. 2012;41:722.

15. Alexander $G R$, et al. A United States national reference for fetal growth. Obstet Gynecol. 1996;87:163.

16. Matijasevich A, et al. Maternal smoking during pregnancy and offspring growth in childhood: 1993 and 2004 Pelotas cohort studies. Arch Dis Child. 2011;96:519-25

17. Bernardi JR, et al. Impact of Perinatal Different Intrauterine Environments on Child Growth and Development in the First Six Months of Life-IVAPSA Birth Cohort: rationale, design, and methods. BMC Pregnancy Childbirth. 2012;12:25.

18. World Health Organization, WHO Child Growth Standards. Methods and development. Length/height-for-age, weight-for-age, weight-for-length, weight-for-height and body mass index-for-age. Geneva: WHO; 2006.

19. National Institute of Science and Technology for Public Policies of Alcohol and Other Drugs. II BNADS Results from the Second Brazilian National Alcohol Survey. Sao Paulo; 2012.

20. Suzuki $K$, et al. Effect of maternal smoking cessation before and during early pregnancy on fetal and childhood growth. J Epidemiol. 2014;24:60.

21. Vardavas $\mathrm{Cl}$, et al. Smoking and smoking cessation during early pregnancy and its effect on adverse pregnancy outcomes and fetal growth. Eur J Pediatr. 2010;169:741.

22. Silveira VM, Horta BL. Birth weight and metabolic syndrome in adults: metaanalysis. Rev Saude Publica. 2008;42:10.

23. Beyerlein A, et al. Is low birth weight in the causal pathway of the association between maternal smoking in pregnancy and higher BMI in the offspring? Eur J Epidemiol. 2011;26:413.

24. Adams AK, Harvey HE, Prince RJ. Association of maternal smoking with overweight at age 3 y in American Indian children. Am J Clin Nutr. 2005;82:393-8.

25. Monasta $L$, et al. Early-life determinants of overweight and obesity: a review of systematic reviews. Obes Rev. 2010;11:695.

26. Sharma AJ, Cogswell ME, Li R. Dose-response associations between maternal smoking during pregnancy and subsequent childhood obesity: effect modification by maternal race/ethnicity in a low-income US cohort. Am J Epidemiol. 2008;168:995.

27. Iliadou AN, et al. Familial factors confound the association between maternal smoking during pregnancy and young adult offspring overweight. Int J Epidemiol. 2010;39:1193.

28. Hiscock R, Bauld L, Amos A, Fidler JA, Munafò M. Socioeconomic status and smoking: a review. Ann N Y Acad Sci. 2012;1248:107.

29. Tong VT, et al. Estimates of Smoking Before and During Pregnancy, and Smoking Cessation During Pregnancy: Comparing Two Population-Based Data Sources. Public Health Rep (1974-). 2013;128;179-88.

30. Harrod CS, et al. Quantity and timing of maternal prenatal smoking on neonatal body composition: the healthy start study. J Pediatr. 2014;165:707-12.

31. Koshy G, Delpisheh A, Brabin BJ. Dose response association of pregnancy cigarette smoke exposure, childhood stature, overweight and obesity. Eur J Pub Health. 2011:21:286.

32. Jones $\mathrm{G}$, Hynes $\mathrm{KL}$, Dwyer $\mathrm{T}$. The association between breastfeeding, maternal smoking in utero, and birth weight with bone mass and fractures in adolescents: a 16-year longitudinal study. Osteoporos Int. 2013;24:1605.

33. Dewey KG. Nutrition, growth, and complementary feeding of the breastfed infant. Pediatr Clin N Am. 2001;48:87.

34. Stettler $\mathrm{N}$, et al. Weight gain in the first week of life and overweight in adulthood: a cohort study of European American subjects fed infant formula. Circulation. 2005;111:1897.

35. Ingvarsson RF, et al. The effects of smoking in pregnancy on factors influencing fetal growth. Acta Paediatr. 2007;96:383.

36. Bartholomeusz HH, Courchesne E, Karns CM. Relationship between head circumference and brain volume in healthy normal toddlers, children, and adults. Neuropediatrics. 2002:33:239.

37. García-alix A, et al. Ability of neonatal head circumference to predict long term neurodevelopmental outcome. Rev Neurol. 2004;39:548-54.

38. Ekelund $U$, et al. Upward weight percentile crossing in infancy and early childhood independently predicts fat mass in young adults: the Stockholm Weight Development Study (SWEDES). Am J Clin Nutr. 2006;83:324-30. 
39. Heaman $M$, et al. Risk factors for preterm birth and small-for-gestational-age births among Canadian women. Paediatr Perinat Epidemiol. 2013;27:54.

40. Reeves S, Bernstein I. Effects of maternal tobacco-smoke exposure on fetal growth and neonatal size. Expert Rev Obstet Gynecol. 2008:3:719.

41. Lambers DS, Clark KE. The maternal and fetal physiologic effects of nicotine. Semin Perinatol. 1996:20:115.

42. Riedel C, et al. Differences in BMI z-scores between offspring of smoking and nonsmoking mothers: A longitudinal study of german children from birth through 14 years of age. Environ Health Perspect. 2014;122:761.

43. Toschke AM, et al. Early intrauterine exposure to tobacco-inhaled products and obesity. Am J Epidemiol. 2003;158:1068-74.

Submit your next manuscript to BioMed Central and we will help you at every step:

- We accept pre-submission inquiries

- Our selector tool helps you to find the most relevant journal

- We provide round the clock customer support

- Convenient online submission

- Thorough peer review

- Inclusion in PubMed and all major indexing services

- Maximum visibility for your research

Submit your manuscript at www.biomedcentral.com/submit
) Biomed Central 